PAPER

\title{
Does the method of treatment of acutely ruptured intracranial aneurysms influence the incidence and duration of cerebral vasospasm and clinical outcome?
}

\author{
A J P Goddard, P P J Raju, A Gholkar
}

J Neurol Neurosurg Psychiatry 2004;75:868-872. doi: 10.1136/jnnp.2003.033068

See end of article for authors' affiliations

.....................

Correspondence to: Dr A Goddard, MRI Department, Imaging Centre, Queens Medical Centre, Derby Road, Nottingham, NG7 2UH, UK; Tony.Goddard@mail. qmc.uh-tr.trent.nhs.uk

Received

21 November 2003

Accepted

21 November 2003

\begin{abstract}
Objectives: Cerebral vasospasm remains the leading cause of death and permanent disability after subarachnoid haemorrhage. This study determined whether the method of aneurysm treatment plays an important role in determining the incidence of cerebral vasospasm and its clinical consequences.

Methods: Admission data, cranial computed tomography (CT), treatment details, transcranial Doppler (TCD) results, and clinical outcomes of patients who had surgical or endovascular management of their ruptured aneurysm were recorded and subject to multivariate analysis.

Results: Between January 1995 and December 1999, 292 eligible patients (206 female, 86 male) had definitive aneurysm treatment at our unit. 212 patients were clipped, 80 coiled. There was no significant difference in patient age, pre-treatment neurological grade, Fisher grade, or timing of treatment in the two groups. $48.3 \%$ patients developed TCD detected cerebral vasospasm and $16.1 \%$ patients developed permanent ischaemic neurological deficit. At clinical follow up, $84.2 \%$ of patients were well (mGOS 1 and 2) with a cumulative death rate of $6.5 \%$ from all causes.

The only significant predictor of TCD-detected cerebral vasospasm was patient age (inversely, $p=0.004$ ). Increased patient age, vasospasm, poor pre-treatment WFNS, and higher CT Fisher grades correlated with a poor discharge GOS. However, only poor pre-treatment WFNS grade and patient age correlated with poor GOS at follow up $(p<0.001)$.

Conclusion: The treatment method had no influence on the incidence or duration of TCD detected vasospasm and there was no significant difference in outcome at discharge or follow up between those patients who had surgery or endovascular management of their aneurysms.
\end{abstract}

\section{MATERIALS AND METHODS}

The case notes, TCD results, cranial computed tomography (CT) examinations, angiograms, and outpatient correspondence were reviewed for all patients who received definitive treatment for ruptured aneurysms from January 1995 to December 1999. During this time a single operator was performing Guglieimi Detachable Coils (GDC) embolisation, an experienced team of neurosurgeons operated on our patient cohort, and a single operator performed TCD analysis.

All patients underwent cranial CT to confirm a clinical diagnosis of SAH. If CT was normal, all patients underwent lumbar puncture to detect CSF xanthochromia unless there was a contraindication. Only patients with unequivocally positive results were included in the analysis. All patients had four-vessel cerebral angiography performed prior to planning treatment.

The method of aneurysm treatment was made on an individual patient basis, depending on clinical grade, aneurysm location and morphology, and co-morbidity. Where appropriate, treatment was randomised as part of the International Subarachnoid Aneurysm Trial (ISAT). All patients were admitted either to intensive care or the high dependency unit for a minimum of 24 hours after the procedure and received equivalent levels of care appropriate to their condition. Oral or intravenous nimodipine was administered to all patients for a minimum of 21 days depending on clinical condition.

Abbreviations: CSF, cerebrospinal fluid; $\mathrm{CT}$, computed tomography; GDC, Guglieimi Detachable colis; ISAT, International Subarachnoid Aneurysm Trial; mGOS, modified Glasgow Outcome Score; SAH, subarachnoid haemorrhage; TCD, transcranial Doppler 
TCD monitoring with a $2 \mathrm{MHz}$ probe commenced at day one and continued on alternate days until death, clinical recovery, or normalisation of velocities. Bilateral pterional insonation was performed on the intracranial internal carotid $\mathrm{Al}$ and Ml vessels. Suboccipital insonation was performed to measure velocities in the basilar artery. Patients were excluded from the analysis if TCD was unable to be performed-for example, absence of an acoustic windowor an incomplete study only was possible.

The presence of vasospasm was defined as a rise above $120 \mathrm{~cm} / \mathrm{s}$ in any insonated vessel. This low velocity threshold increases sensitivity and allows preventative treatment to be given. Development of focal neurological deficit or deterioration in conscious level was noted. In the setting of neurological deterioration, cranial CT was performed to exclude non-ischaemic causes-for example, post-operative haematoma, re-bleed, and hydrocephalus. Patients having a non-ischaemic cause for their deterioration continued to be monitored and only a deficit directly attributable to ischaemia was recorded.

Standard haemodilution, hypertension, and hyperhydration (triple-H) treatment was given if the patient showed TCD or clinical signs of cerebral vasospasm. This was continued along with other medical treatments as appropriate until the patient recovered, died, or TCD velocities normalised. If the patient continued to deteriorate despite intensive triple- $\mathrm{H}$ treatment, then consideration was given to performing papaverine infusion directly into the affected vessels.

Specific complications related to treatment or significant systemic complications of SAH were noted.

Mortality and modified Glasgow Outcome Score (mGOS) were recorded for all patients at discharge and follow up 4-8 months post-treatment. Patients were excluded from analysis if there was incomplete follow up data.

\section{RESULTS}

\section{Pre-treatment patient characteristics}

A total of 292 patients satisfied the selection criteria for analysis. Age, sex, pre-treatment World Federation of Neurological Surgeons (WFNS) grade, CT Fisher grade, aneurysm location, time between haemorrhage and treatment, presence of vasospasm, ischaemic neurological deficit, discharge, and follow up mGOS were recorded and these data are presented in table 1. Additional data recorded included duration of vasospasm and specific complications. Statistical analysis was performed with commercial software (SPSS for Windows, Release 7.7.1).

Mantel-Haenszel $\chi^{2}$ analysis for differences in trends showed significantly higher CT Fisher grade patients receiving endovascular treatment $(p=0.044)$. There was a nonsignificant predominance of posterior circulation aneurysms and poorer grade patients in the endovascular group $(p=0.18)$. There was no significant difference in patient age or sex in the two groups.

A total of 220 aneurysms were clipped in 212 patients and 80 aneurysms in 80 patients were coiled. Seven patients had two aneurysms clipped at one sitting; one patient had a second aneurysm clipped 2 days after the first. In these cases, there was doubt as to which aneurysm had ruptured. One patient had an anterior choroidal aneurysm coiled then subsequently clipped, one had an anterior communicating artery aneurysm clipped and wrapped, and one had a small carotid-ophthalmic aneurysm wrapped.

\section{Incidence of TCD detected cerebral vasospasm}

Thirty eight out of $80(47.5 \%)$ of coiled and 103/212 (48.6\%) of clipped patients (141/292 [48.3\%] overall) developed TCD velocities above $120 \mathrm{~cm} / \mathrm{s}$. The characteristics of those patients who did and did not develop TCD vasospasm and those who developed ischaemic neurological deficit are shown in table 1. Pearson $\chi^{2}$ and Mantel-Haenszel $\chi^{2}$ analysis of the following variables showed no correlation with the incidence of vasospasm: method of treatment $(\mathrm{p}=0.696)$, patient sex $(\mathrm{p}=0.923)$, aneurysm site $(p=0.892)$, WFNS grade $(p=0.496)$, and CT Fisher grade $(\mathrm{p}=0.932)$.

Multiple logistic regression analysis confirmed that the only independent factor correlating with the presence of TCD detected vasospasm was young patient age $(p=0.0037)$.

Ninety one $(64.5 \%)$ patients had treatment prior to onset of spasm, $32(22.7 \%)$ had treatment after vasospasm onset, and $18(12.8 \%)$ had treatment on the same day spasm was demonstrated. There was no difference in treatment method in these groups. Two patients underwent papaverine infusion to allow endovascular aneurysm treatment. Four patients had papaverine infusion after their aneurysms had been clipped. All of these patients showed significant TCD velocity rises, indicating good correlation between TCD findings and angiography in this group.

There was no significant difference in the proportion of patients in either treatment group who suffered ischaemic neurological deficit (Pearson $\chi^{2}: p=0.69$ ) or in the groups of patients who had TCD velocity rises $(\mathrm{p}=0.089)$.

There was no difference in the duration of vasospasm in two groups (Mann-Whitney $U$ test: $p=0.975$ ). Mean spasm duration was 2.9 days (95\% confidence interval [CI]: 1.3 to 4.5 ) and 2.88 days (95\% CI: 2.08 to 3.68 ) in patients with and without delayed ischaemic neurologic deficit (DIND), respectively.

\section{Patient outcomes}

Nineteen out of $292(6.5 \%)$ patients died in hospital: 18/19 $(95 \%)$ as a direct result of SAH or treatment and one died due to systemic complications. Eight out of 19 (42\%) patients developed TCD velocities indicative of vasospasm; not significantly different from our overall patient population.

Table 1 documents the mGOS of the patients at the time of discharge and follow up. A total of 221/292 (75.7\%) patients were well (mGOS I and II) at discharge, which increased to $246 / 292(84 \%)$ at follow up. The capacity for recovery was directly related to discharge mGOS: $66 \%$ of mGOS II, $74 \%$ of mGOS III, and $40 \%$ of mGOS IV patients improved at follow up. There was no difference in functional recovery between coiled and clipped patients who developed TCD velocity rises and neurological deficit.

Seven patients died during the follow up period: five succumbed to complications of their haemorrhage and two died of unrelated causes. Multivariate analysis of factors influencing outcome at discharge and follow up is summarised in table 2.

Six patients re-bled prior to surgical (four patients) or endovascular (two patients) treatment. Five out of six patients $(83 \%)$ developed vasospasm and two of these patients died. Two patients were vegetative (mGOS 4) and two were mGOS 2. Eight patients suffered intraprocedural aneurysm rupture (six surgical, two endovascular). Three of these patients died soon after and three of the remaining five patients $(60 \%)$ developed vasospasm and were mGOS 1, 2, and 3 at follow up. The two patients who did not develop vasospasm were mGOS 2 and 3 at follow up. One patient died because of early re-rupture after coiling of an anterior communicating artery aneurysm. Preprocedure and periprocedure re-rupture were significant predictors of a poor outcome in both groups with a mortality and major morbidity (mGOS 4 and 5 ) of $50 \%$ and were responsible for five out of $19(26 \%)$ of all deaths $(\mathrm{p}<0.001)$. 
Table 1 Patient demographics

\begin{tabular}{|c|c|c|c|c|c|}
\hline & Overall & Surgical & Endovascular & TCD vasospasm & No spasm \\
\hline \multicolumn{6}{|l|}{ Age (years) } \\
\hline Range & $18-84$ & $18-84$ & 19-82 & & \\
\hline Mean & 53.7 & 53.7 & 54.0 & 53.6 & 53.8 \\
\hline $95 \% \mathrm{Cl}$ & $52.1-55.3$ & $51.8-55.6$ & $50.9-57.1$ & $51.3-56.0$ & $51.5-56.1$ \\
\hline \multicolumn{6}{|l|}{ Sex } \\
\hline$M(\%)$ & 85 & $64(31.1)$ & $21(26.3)$ & $41 / 141(29.1)$ & $\begin{array}{l}45 / 151 \\
(29.8)\end{array}$ \\
\hline$F(\%)$ & 203 & $144(68.9)$ & $59(73.7)$ & $100 / 141(70.9)$ & $\begin{array}{l}106 / 151 \\
(70.2)\end{array}$ \\
\hline \multicolumn{6}{|c|}{ Aneurysm location (\%) } \\
\hline A com & $107(35.7)$ & $74(33.6)$ & $33(41.3)$ & & \\
\hline $\mathrm{P}$ com & $83(27.7)$ & $59(26.8)$ & $24(30)$ & & \\
\hline MCA & $66(22)$ & $58(26.4)$ & $8(10)$ & & \\
\hline Car ophth & $6(2)$ & $4(1.8)$ & $2(2.5)$ & & \\
\hline ICA other & $19(6.3)$ & & $13(5.9)$ & $6(7.5)$ & \\
\hline Pericallosal & $7(2.3)$ & & $7(3.2)$ & $0(0)$ & \\
\hline Post cerebral A & $2(0.7)$ & $1(0.45)$ & $1(1.3)$ & & \\
\hline PICA & $4(1.3)$ & $1(0.45)$ & $3(3.7)$ & & \\
\hline BA & $6(2)$ & $3(1.4)$ & $3(3.7)$ & & \\
\hline \multicolumn{6}{|l|}{ Fisher grade (\%) } \\
\hline I & $33(11.4)$ & $28(13.4)$ & $56.2)$ & $15(10.6)$ & $18(11.9)$ \\
\hline$\|$ & $91(31.5)$ & 69 (33) & $22(27.5)$ & $43(30.5)$ & 48 (31.8) \\
\hline III & $87(30.1)$ & $64(30.6)$ & $23(28.8)$ & $42(29.8)$ & $47(31.1)$ \\
\hline IV & 78 (27) & $48(23)$ & 30 (37.5) & $41(29.1)$ & $38(25.2)$ \\
\hline \multicolumn{6}{|l|}{ WFNS grade (\%) } \\
\hline I & $126(43.6)$ & $96(45.9)$ & $30(37.5)$ & $64(45.4)$ & $65(43)$ \\
\hline$\|$ & $69(23.9)$ & $50(24)$ & $19(23.8)$ & $27(19.1)$ & $42(27.8)$ \\
\hline III & $50(17.3)$ & 37 17.7) & $13(16.2)$ & $26(18.4)$ & $24(15.9)$ \\
\hline IV & $38(13.1)$ & $23(11.0)$ & $15(18.8)$ & $21(15)$ & $17(11.3)$ \\
\hline V & $6(2.1)$ & $3(1.4)$ & $3(3.7)$ & $3(2.1)$ & $3(2)$ \\
\hline \multicolumn{6}{|c|}{ Time between ictus and Rx (days) } \\
\hline Range & $0-52$ & $0-52$ & $0-33$ & & \\
\hline Mean & 5.45 & 5.46 & 5.54 & 5.45 & 5.45 \\
\hline $95 \% \mathrm{Cl}$ & $4.7-6.2$ & $4.5-6.4$ & $4.0-7.1$ & $4.3-6.6$ & $4.4-6.6$ \\
\hline \multicolumn{6}{|c|}{ Ischaemic neurological deficit } \\
\hline Yes & $47 / 292(16.1)$ & $35 / 212(16.5)$ & $11 / 80(13.8)$ & $29 / 141(20.6)$ & $\begin{array}{l}19 / 151 \\
(12.6)\end{array}$ \\
\hline No & $245 / 292$ (83.9) & ) $177 / 121$ (83.5) & $69 / 80(86.2)$ & $113 / 141(79.4)$ & $\begin{array}{l}132 / 151 \\
(87.4)\end{array}$ \\
\hline \multicolumn{6}{|c|}{ Modified GOS discharge } \\
\hline 1 & $114(39.1)$ & $82(38.7)$ & $32(40)$ & & \\
\hline ॥ & $107(36.6)$ & $85(40.1)$ & $22(27.5)$ & & \\
\hline III & $37(12.7)$ & $23(10.8)$ & $14(17.5)$ & & \\
\hline IV & $15(5.1)$ & $9(4.3)$ & $6(7.5)$ & & \\
\hline V & $19(6.5)$ & $13(6.1)$ & $6(7.5)$ & & \\
\hline \multicolumn{6}{|l|}{ Follow up } \\
\hline 1 & $187(64)$ & $134(63.2)$ & $53(66.25)$ & & \\
\hline$\|$ & $59(20.2)$ & $50(23.6)$ & $9(11.25)$ & & \\
\hline III & $13(4.5)$ & $7(3.3)$ & $6(7.5)$ & & \\
\hline IV & $7(2.4)$ & $4(1.9)$ & $3(3.75)$ & & \\
\hline $\mathrm{V}$ & $26(8.9)$ & $17(8) 9$ & $(11.25)$ & & \\
\hline
\end{tabular}

$\mathrm{TCD}$, transcranial Doppler; $\mathrm{Cl}$, confidence interval; $\mathrm{A}$ com, anterior communicating artery; $\mathrm{P}$ com, posterior communicating artery; MCA, middle cerebral artery; car ophth, carotid ophthalmic; ICA, internal carotid artery; PICA, posterior inferior cerebellar artery; BA, basilar artery; WFNS, World Federation of Neurological Surgeons; Rx, treatments; GOS, Glasgow Outcome Score.

Table 2 Multivariate analysis of the correlation between clinical and treatment parameters and patient outcome at discharge and follow up

\begin{tabular}{lll}
\hline & Discharge GOS & Outpatient GOS \\
\hline Age & $S(p<0.001)$ & $S(p<0.001)$ \\
Sex & $N S(p=0.178)$ & $N S(p=0.34)$ \\
Aneurysm site & $N S(p=0.089)$ & $N S(p=0.523)$ \\
Treatment & $N S(p=0.160)$ & $N S(p=0.102)$ \\
$\begin{array}{l}\text { Method } \\
\text { Timing of treatment }\end{array}$ & $N S(p=0.128)$ & $N S(p=0.108)$ \\
Fisher grade & $S(p=0.0014)$ & $N S(p=0.139)$ \\
WFNS grade & $S(p<0.001)$ & $S(p<0.001)$ \\
TCD velocity & $S(p=0.012)$ & $N S(p=0.284)$ \\
$>120 \mathrm{~cm} / \mathrm{s}$ & $S(p=0.5$
\end{tabular}

GOS, Glasgow Outcome Score; NS, not significant; S, significant; TCD, transcranial Doppler.

\section{DISCUSSION}

Cerebral vasospasm is a significant determinant of patient outcome even after technically successful aneurysm treatment. Vasospasm affects between $30 \%$ and $70 \%$ of patients and leads to clinically significant neurological deficit in $30 \%$ of survivors. ${ }^{3-6}$ The aetiology of this phenomenon has been much studied but remains only partially understood. It would seem that both subarachnoid blood and manipulation of the brain and vessels could lead to vasospasm.

The incidence of symptomatic vasospasm after endovascular treatment of aneurysms has been reported between $19 \%$ and $23 \% .^{14-16}$ This compares well with surgical series. ${ }^{3-5}$ 17-19 Our clinical results also correlate with these series with ischaemic neurological deficit occurring in $13.8 \%$ of endovascular and $16.5 \%$ of surgical patients.

Yalamanchilli et al $^{16}$ in 37 patients ( 19 clipped, 18 coiled) demonstrated significant reduction in spasm in the 
endovascular group compared with surgery. The reasons are likely to be multifactorial, but vessel manipulation, diuresis, and release of blood and prostaglandins as a result of surgery probably outweigh any potential benefit of CSF washout and irrigation with thrombolytic or papaverine.

The largest study into the effects of aneurysm treatment on the incidence of vasospasm was reported by Charpentier et al. ${ }^{15}$ in 244 patients. Their retrospective data included more endovascular cases ( $145 \vee 99$ surgical) but reached similar conclusions; there is no significant difference in the incidence of cerebral vasospasm in surgical $(22.2 \%)$ or endovascular (17.2\%) groups, and young age and treatment complications were a significant predictor of vasospasm occurrence. They also showed a significant correlation between good neurological grade and occurrence of vasospasm, although we demonstrated no such association.

Their study also focused on the important impact of secondary brain insults, such as hyperglycaemia in exacerbating neurological damage. Pre-existing medical conditions and secondary insults undoubtedly contribute in individual cases and explain the poorer outcomes in the elderly across all clinical grades.

The sensitivity of TCD for the detection of cerebral vasospasm in the anterior cerebral circulation ranges from $53 \%$ in the anterior communicating artery to $93 \%$ in the middle cerebral artery ${ }^{20-23}$ with specificity up to $100 \%$ in the anterior and middle cerebral arteries. ${ }^{22}$ TCD of the posterior circulation yields a sensitivity and specificity of $44.0 \%$ and $87.5 \%$ for vertebral and $76.9 \%$ and $79.3 \%$ for basilar artery vasospasm, ${ }^{24}$ although its use here is less widespread.

A velocity reading of $120 \mathrm{~cm} / \mathrm{s}$ may be regarded as a significant rise and a predictor of the presence of angiographic vasospasm. ${ }^{25}$ However, several studies, including our own, have been unable to establish a direct correlation between absolute TCD velocities and the onset of ischaemic neurological deficit. ${ }^{26-29}$ There are technical and physiological reasons why this may be so. A rise in TCD velocities may not indicate vasospasm in the setting of impaired cerebral autoregulation $^{30}$ when increases in circulating volume and hypertension (induced by triple- $\mathrm{H}$ treatment) increase cerebral blood flow sufficiently to be detected as a TCD velocity rise. The expected velocity increases due to vasospasm may be dampened in the setting of raised intracranial pressure. ${ }^{31}$ This is particularly so when mean velocities are used. Patients with systemic hypertension may not demonstrate the rise in TCD velocities expected with the onset of vasospasm. $^{32}$ Insonated vessels may demonstrate normal velocity if flow is dampened by proximal focal spasm. ${ }^{29}$ Additionally, complex flow patterns in patients with SAHrelated neurological deterioration have been demonstrated on positron emission tomography suggesting combined hyperperfusion and vasospasm in some cases, which would clearly cause misleading TCD results. ${ }^{29}$

Although TCD is sensitive to spasm in proximal vessels, vasospasm affects distal vessels also and TCD results may not reflect the severity of spasm in these territories and therefore may not predict clinical outcome. Another possible explanation for the lack of clinical correlation between high TCD velocities and neurological deficit is that the institution of therapeutic measures in response to such TCD velocity rises (particularly triple-H treatment) is effective in averting DIND. Our data do not allow us to test these hypotheses, but it is likely to be a combination of factors.

TCD detected proximal vessel velocity increase is only part of the physiological and clinical profile of the condition of cerebral vasospasm and ischaemia. Defining cerebral vasospasm by relatively low velocity measurements may include patients who do not have vasospasm at all. However, the distribution of false positive results is not expected to be different between the two treatment groups and would not be expected to affect the validity of our findings. High TCD velocities associated with neurological deficit clearly indicate vasospasm, but the opportunity to institute effective treatment may have passed if one accepts these more specific criteria for diagnosis.

Discharge mGOS was directly related to the effects of the initial haemorrhage, patient age, and the presence of high TCD velocities. There was no correlation with the treatment method.

Preprocedural and periprocedural re-rupture was a significant predictor of a poor patient outcome with an $83 \%$ incidence of TCD detected vasospasm and a poor outcome (mGOS 5/5) in 7/14 (50\%) patients.

GOS may be impaired in patients who have no focal deficit—for example, neuropsychological disability—which may at least in part explain the lack of correlation with the presence of vasospasm. Elderly patients and those significantly damaged by their initial haemorrhage (whether or not they re-rupture) have a poor outcome that is not explained by the presence of vasospasm.

Although younger age was associated with a higher vasospasm risk, the outcome in the elderly with vasospasm was poorer. Intact vasoreactivity, efficient collateral flow, or improved cerebral reserve in young patients may all be factors operating to explain this phenomenon.

Vasospasm duration was not a predictor of outcome. It has been previously demonstrated that a significant rise in velocities may predict ischaemic neurological deficit. ${ }^{33}$ A sustained but modest increase in TCD velocity (shown in some of our patients) may be tolerated as collateralisation has time to become established and lessen the impact on overall cerebral perfusion. Chronic vessel wall changes after vasospasm may be responsible for persistent velocity abnormalities in the face of a clinically well patient.

The ISAT demonstrated a $22.6 \%$ relative, $6.9 \%$ absolute reduction in poor outcomes between endovascularly and surgically treated aneurysms. ${ }^{34}$ Our study suggests that this difference is not explained by differences in the incidence or severity of vasospasm, which is the same in the two treatment groups. Measures to more effectively monitor and reduce the clinical impact of vasospasm will improve patient outcomes even further.

\section{CONCLUSION}

Endovascular aneurysm treatment has no adverse impact on the occurrence of TCD detected vasospasm, on the incidence of ischaemic neurological deficit, or on overall patient outcome. The only factor that predicted the presence of vasospasm was young patient age. Factors correlating with poor patient outcomes were increasing age and admission WFNS grade. TCD correlates poorly with patient outcome.

\section{Authors' affiliations}

A J P Goddard, P P J Raju, A Gholkar, Neurosciences Department, Newcastle General Hospital, Westgate Road, Newcastle-upon-Tyne, NE4 6BE, UK

A J P Goddard, MRI Department, Imaging Centre, Queens Medical Centre, Derby Road, Nottingham NG7 2UH, UK

Competing interests: none declared

\section{REFERENCES}

1 Kassel NF, Torner JC, Haley EC Jr, et al. The International Cooperative Study on the Timing of Aneurysm Surgery. Part 1: Overall management results. J Neurosurg 1990;73:18-36.

2 Kassel NF, Torner JC, Jane JA, et al. The International Cooperative Study on the Timing of Aneurysm Surgery. Part 2: Surgical results. J Neurosurg 1990;73:37-47.

3 Adams HP Jr, Kassell NF, Torner JC, et al. Predicting outcome ischaemia after aneurysmal subarachnoid haemorrhage: influences of clinical condition, CT 
results, and antifibrinolytic therapy: a report of the Co-operative Aneurysm Study. Neurology 1987;37:1586-91.

4 Rabb CH, Tang G, Chin LS. A statistical analysis of factors related to symptomatic cerebral vasospasm. Acta Neurochir 1994;127:27-31.

5 Solomon RA, Onesti ST, Klebanoff L. Relationship between the timing of aneurysm surgery and the development of delayed cerebral ischaemia. J Neurosurg 1991;75:56-61

6 Biller J, Godersky JC, Adams HP Jr. Management of aneurysmal subarachnoid haemorrhage. Stroke 1988:19:1300-5.

7 MacDonald RL, Weir BK. A review of haemoglobin and the pathogenesis of cerebral vasospasm. Stroke 1991;22:971-82.

8 Osaka K. Prolonged vasospasm produced by the breakdown products of erythrocytes. J Neurosurg 1977:47:403-11.

9 Handa Y, Weir BK, Nosko M, et al. The effect of timing of clot removal on chronic vasospasm in a primate model. J Neurosurg 1987;67:558-64.

10 Auer LM, Mokry M. Disturbed cerebrospinal fluid after subarachnoid haemorrhage and acute aneurysm surgery. Neurosurg 1990:26:804-9.

11 Wardlaw JM, Offin R, Teasdale GM, et al. Is routine transcranial Doppler ultrasound monitoring useful in the management of subarachnoid haemorrhage? I Neurosurg 1998;88:272-6.

12 Zygmunt SC, Delgado-Zygmunt TJ. The haemodynamic effect of transcranial Doppler-guided high dose nimodipine treatment in established vasospasm after subarachnoid haemorrhage. Acta Neurochir 1995; 135:179-85

13 Hurst RW, Schnee C, Raps EC, et al. Role of transcranial Doppler in neuroradiological treatment of intracranial vasospasm. Stroke 1993;24:299-303.

14 Murayama Y, Malisch T, Guglielmi G, et al. Incidence of cerebral vasospasm after endovascular treatment of acutely ruptured aneurysms: report on 69 cases. J Neurosurg 1997;87:830-5.

15 Charpentier C, Audibert G, Guillemin F. Multivariate analysis of predictors of cerebral vasospasm occurrence after aneurysm subarachnoid haemorrhage. Stroke 1999;30:1402-8

16 Yalamanchilli K, Rosenwasser RH, Thomas JE, et al. Frequency of cerebral vasospasm in patients treated with endovascular occlusion of intracranial aneurysms. Am J Neuroradiol 1998;19:553-8.

17 Haley EC Jr, Kassel NF, Torner JC, et al. A randomized trial of two doses of Nicardipine in aneurysmal subarachnoid haemorrhage: a report of the cooperative aneurysm study. J Neurosurg 1994;80:788-96.

18 Ingawa T, Kamaika K, Matsuda Y. Effect of continuous cisternal drainage on cerebral vasospasm. Acta Neurochir 1991;112:28-36.

19 Miyaoka M, Sato K, Ishii S. A clinical study of the relationship of timing to outcome of surgery for ruptured cerebral aneurysms: a retrospective analysis. J Neurosurg 1993;79:373-8.

20 Creissard $\mathrm{P}$, Proust $\mathrm{F}$, Langlois $\mathrm{O}$. Vasospasm diagnosis: theoretical and real transcranial Doppler sensitivity. Acta Neurochir 1995;136:181-5.
21 Creissard P, Proust F. Vasospasm diagnosis: theoretical sensitivity of transcranial Doppler evaluated using 135 angiograms demonstrating vasospasm. Practical consequences. Acta Neurochir 1994;131:12-18.

22 Sloan MA, Haley EC Jr, Kassell NF, et al. Sensitivity and specificity of transcranial Doppler ultrasonography in the diagnosis of vasospasm following subarachnoid haemorrhage. Neurology 1989;39:1514-18.

23 Langlois $\mathrm{O}$, Rabehenoina $C$, Proust $F$, et al. Diagnosis of vasospasm: comparison between arteriography and transcranial Doppler. A series of 112 comparative tests. Neuro-Chirurgie 1992;38:138-40.

24 Sloan MA, Burch CM, Wozniak MA, et al. Transcranial Doppler detection of vertebrobasilar vasospasm following subarachnoid haemorrhage. Stroke 1994:25:2187-97.

25 Newell DW, Winn HR. Transcranial Doppler in cerebral vasospasm. Neurosurg Clin North Am 1990;1:319-28.

26 Grosset DG, Straiton J, McDonald I, et al. Use of transcranial Doppler sonography to predict development of delayed ischaemic deficit after subarachnoid haemorrhage. I Neurosurg 1993;78:183-7.

27 Laumer R, Steinmeier R, Gonner F, et al. Cerebral haemodynamics in subarachnoid haemorrhage evaluated by transcranial Doppler sonography. Part 1: Reliability of flow velocities in clinical management. Neurosurg 1993:33:1-8.

28 Ekelund A, Saveland H, Romner B, et al. Is transcranial Doppler sonography useful in detecting late cerebral ischaemia after aneurysmal subarachnoid haemorrhage? Br J Neurosurg 1996;10:19-25.

29 Minhas PS, Menon DK, Smielewski P, et al. Positron emission tomographic cerebral perfusion disturbances and transcranial Doppler findings among patients with neurological deterioration after subarachnoid haemorrhage. Neurosurg 2003;52(5):1017-22.

30 Manno EM, Gress DR, Schwann LH, et al. Effects of induced hypertension on transcranial Doppler ultrasound velocities in patients after subarachnoid haemorrhage. Stroke 1998;29:422-8.

31 Klingelhöfer J, Sander D, Holzgraeffe M, et al. Cerebral vasospasm evaluated by transcranial Doppler ultrasonography at different intracranial pressures. J Neurosurg 1991;75:752-8.

32 Ekelund A, Saveland H, Romner B, et al. Transcranial Doppler ultrasound in hypertensive versus normotensive patients after aneurysmal subarachnoid haemorrhage. Stroke 1995:26:2071-4.

33 Grosset DG, Straiton J, du Trevou M, et al. Prediction of symptomatic vasospasm after subarachnoid haemorrhage by rapidly increasing transcranial Doppler velocity and cerebral blood flow changes. Stroke 1992;23:674-9.

34 Molyneux A, Kerr R, Stratton I, International Subarachnoid Aneurysm Trial (ISAT) Collaborative Group. International Subarachnoid Aneurysm Trial (ISAT) of neurosurgical clipping versus endovascular coiling in 2143 patients with ruptured intracranial aneurysms: a randomised trial. Lancet 2002;360(9342):1267-74.

\section{$\mathrm{ECHO}$}

\section{Value of serum prolactin in the management of syncope}

\section{S Ahmad, M W Beckett}

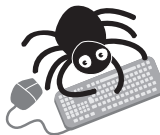

Please visit the Journal of Neurology, Neurosurgery, and Psychiatry website [www.jnnp. com] for a link to the full text of this article.
Objective: A meta-analysis of studies of the usefulness of raised serum prolactin in diagnosing generalised tonic-clonic seizures (GTCS) in patients presenting to the accident and emergency (A\&E) department after a single episode of syncope.

Methods: A three part question was defined. Medline, EMBASE, PubMed, the Cochrane Library were searched to identify relevant studies. Studies were evaluated for eligibility and quality and data extracted to calculate sensitivity (SN), specificity (SP), and likelihood ratios (LR).

Results: Of 13 relevant studies only three met the criteria for evaluation. If a serum prolactin concentration is greater than three times the baseline when taken within one hour of syncope, then in the absence of test "modifiers": (1) the patient is nine times more likely to have suffered a GTCS as compared with a pseudoseizure positive LR $=8.92$ (95\% CI ( 1.31 to $60.91)), \mathrm{SN}=0.62(95 \% \mathrm{CI}(0.40$ to 0.83$)), \mathrm{SP}=0.89(95 \% \mathrm{CI}(0.60$ to 0.98$))$ and (2) five times more likely to have suffered a GTCS as compared with non-convulsive syncope positive LR 4.60 (95\% CI ( 1.25 to 16.90$)$ ), $\mathrm{SN}=0.71$ (95\% CI (0.49 to 0.87$)$ ), SP $=0.85$ (95\% CI $(0.55$ to 0.98$))$.

Conclusion: A positive test result is highly predictive of a GTCS, however a negative test result does not necessarily exclude a seizure. Serum prolactin should be measured in patients presenting to the A\&E department within an hour of a syncopal episode, unless the cause is immediately obvious.

A Emergency Medicine Journal 2004;21:e3 (http://www.emjonline.com/cgi/content/full/21/2/e3). 